\section{Into the depths of ataxia}

\author{
Harry T. Orr \\ Institute of Human Genetics, Department of Laboratory Medicine and Pathology, \\ University of Minnesota, Minneapolis, Minnesota, USA
}

\begin{abstract}
Ataxia is a lethal neurological disease characterized by incoordination, postural abnormalities, difficulties with gait, and problems with clarity of speech. The etiology of ataxia is divided equally between hereditary and sporadic forms. Regardless of cause, the cerebellar cortex is often a target in ataxia. Thus, how a disruption in cerebellar cortex might lead to ataxia is of considerable interest. A report in this issue of the JCI links ataxia to enhanced hyperexcitability of neurons in the deep cerebellar nuclei (see the related article on page 582).
\end{abstract}

J. Clin. Invest. 113:505-507 (2004). doi:10.1172/JCI200421092.

Since the pioneering works of the neuroanatomist and Nobel laureate Santiago Ramón y Cajal, neuroscientists have marveled at the elegant beauty and organizational simplicity of the cerebellar cortex. In fact it was the cerebellum that gave Ramón y Cajal the first indications that favored his neuron doctrine as the basis for organization of the CNS (1). It has long been appreciated that as the sole source of output from the cerebellar cortex, Purkinje cells have a critical role in cerebellar function and its impact on motor performance. Purkinje cells are inhibitory neurons whose major projection is to neurons within the deep cerebellar nuclei (DCN). A link between the excitability of Purkinje cell target neurons in the DCN and proper motor function was established in a well-studied form of motor learning, the conditioned eye blink response (2). In this paradigm, the animal learns to associate a conditioned response, an eye blink, with an auditory tone that normally would not elicit an eye blink. During the learning

\footnotetext{
Address correspondence to: Harry T. Orr, Institute of Human Genetics, Mayo Mail Code 206, University of Minnesota, Minneapolis, Minnesota 55455, USA. Phone: (612) 625-3647; Fax: (612) 626-7031; E-mail: harry@lenti.med.umn.edu.

Conflict of interest: The author has declared that no conflict of interest exists.

Nonstandard abbreviations used: deep cerebellar nuclei (DCN); small-conductance calcium-activated potassium (SK).
}

phase the animal learns to associate the tone with a noxious stimulus, a puff of air to the eye. After a period of learning, the tone alone elicits an eye blink. At the cellular level, the inhibitory signal from Purkinje cells to the DCN is decreased in response to the tone (3). This decrease then permits an increase in DCN firing rate, which initiates the eye blink. Importantly, in this normal physiological process, changes in Purkinje cell and DCN firing rates are transient. In this issue of the JCI, Shakkottai et al. explain that they hypothesized that perhaps a consistent increase in DCN excitability underlies the pathological state of cerebellar ataxia (4). In the case of a cerebellar ataxia, by whatever the cause, Purkinje cell function would be compromised and its inhibitory signal to neurons in the DCN would decrease, which in turn would increase DCN excitability.

To test this hypothesis directly, Shakkottai et al. (4) increased the firing rate of DCN neurons in mice using a transgenic strategy. DCN cells, like many other neurons, express smallconductance calcium-activated potassium (SK) channels. A previous study by Aizenman and Linden (5) using an SK inhibitor indicated that inactivation of these channels increased DCN firing rate. Shakkottai and colleagues took advantage of a truncated SK3-1B transcript. This transcript encodes an SK protein that inhibits all SK chan- nels (6). However, the absence of a DCN-specific promoter complicated the use of a transgenic approach. To overcome this it was necessary to use a regulatory region from the Thy1.2-SX gene that is neuron-specific. While this Thy1.2-SX regulatory region can express strongly in the DCN, it also typically drives expression widely throughout the CNS. The investigators were hoping that the capricious nature of the transgenic approach would act in their favor and a transgenic line with an expression pattern limited to the DCN would be forthcoming. In any case, the investigators would have to be particularly vigilant in their analysis of transgene expression in any ataxic mice generated.

Shakkottai et al. (4) had the foresight to tag the SK3-1B protein with GFP. This enabled them to use immunohistochemistry with antibodies to GFP to examine the cellular pattern of SK3-1B expression in the ataxic mice generated. Relying heavily on the conclusion that the ataxia seen in these mice was of cerebellar origin, their analysis of transgene expression focused on the cerebellum where SK3-1B expression was detected only in DCN neurons. While it is likely that the ataxia is of cerebellar origin, if there is one concern it is the extent to which transgene expression in other regions of the brain might be contributing to the ataxic phenotype. For example, in all four lines with severe ataxia in addition to robust transgene expression in the DCN, expression was seen in the red nucleus (to which the DCN projects), the pontine nucleus (which projects to the DCN), and the motor cortex. In concluding that alterations in the DCN underlie the ataxia, Shakkottai et al. rely on two additional points. First, strong expression was seen in the red nucleus in one mouse line that was not ataxic. This does show that expression in the red nucleus alone was not sufficient to produce the ataxia, but does not formally rule out that expression in the red nucleus does not contribute to the phenotype. Second, the level of transgene expression in the pontine nucleus and motor cortex was 


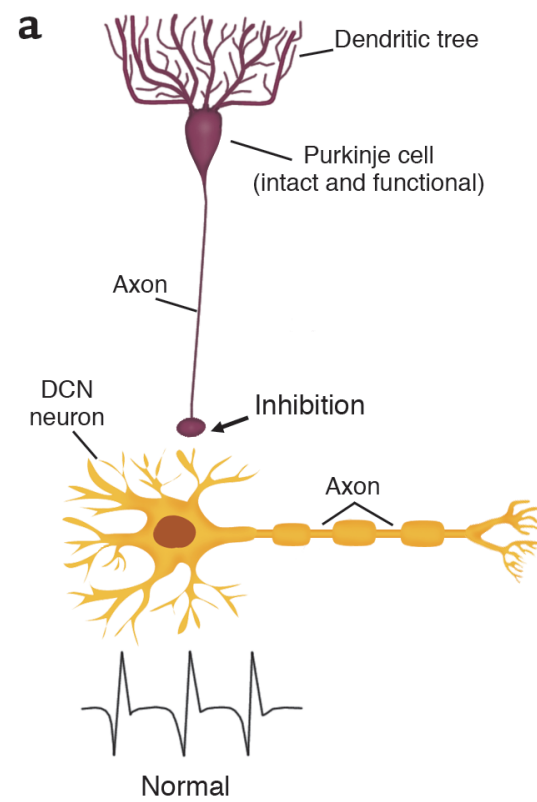

Norma
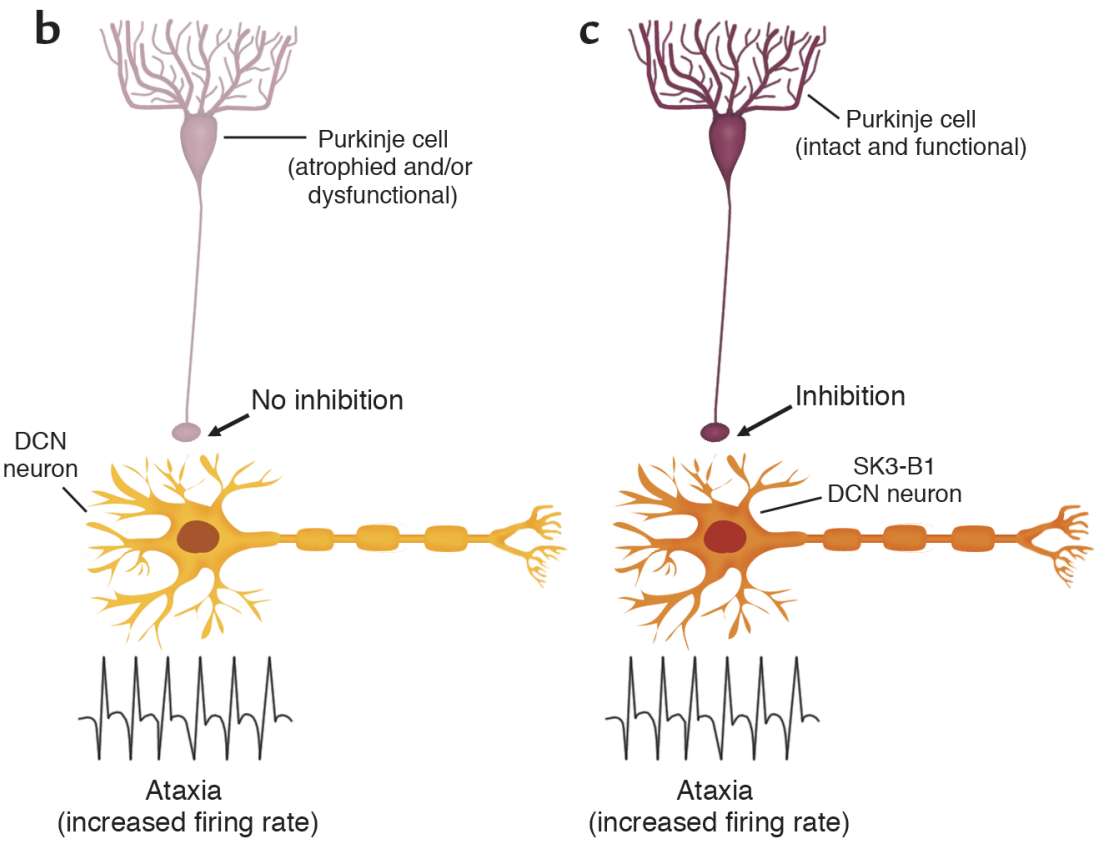

\section{Figure 1}

SK3-B1 mice demonstrate a direct relationship between DCN firing rate and proper movement control. In the normal state (a), Purkinje cells in the cerebellar cortex provide an inhibitory signal to DCN neurons, which, in conjunction with other pathways into the DCN, results in a normal DCN firing rate and proper motor control. In cerebellar ataxia (b), the inhibitory signal to the DCN neurons from Purkinje cells is compromised. This lack of a proper inhibition was suggested to result in an increased firing rate of DCN neurons, leading to ataxia. (c) The direct relationship between increased DCN firing rate and ataxia was demonstrated by Shakkottai et al. (4). When the SK inhibitor SK3-B1 was expressed in DCN neurons, those neurons had an increased firing rate and the transgenic mice were ataxic. Importantly, the Purkinje cell input into the DCN remained intact in the SK3-B1 mice.

judged to not correlate with the severity of the ataxia. Even though the authors provided an excellent illustration of the immunohistochemical scale used to assess expression levels (ref. 4, Figure 5b), some caution is warranted when the level of transgene expression is assessed solely by immunohistochemistry, which is relatively nonquantitative. Regardless, Shakkottai et al. very nicely demonstrated that SK3-1B expression in DCN neurons correlates with an increase in their firing rate consistent with an increase in firing rate seen when SK channels were exposed to a pharmacological inhibitor. Importantly, the observed changes in DCN firing rate in the SK3-1B mice occurred in the absence of any detectable alterations in several other electrical parameters of DCN neurons. The authors went on to show convincingly that the changes in DCN firing rate occurred in the absence of any signs of pathology or degeneration in the cerebellar cortex, as well as elsewhere in the brain.

\section{Some thoughts}

Shakkottai et al. (4) close by suggesting that the drug riluzole, which has the ability to open neuronal SK channels in tissue culture cells (7), may be of therapeutic advantage for ataxia. It is advisable to be cautious in generalizing these data to clinical ataxias as a whole. Suggesting that use of an SKopener, and riluzole specifically, could be beneficial seems premature. First, the pathological picture of the human cerebellar ataxias is exceedingly complex (8). For example, in some ataxias such as spinocerebellar ataxia type 1, both Purkinje cells and DCN neurons are affected. In others, like dentatorubropallidoluysial atrophy, DCN neurons are affected in the apparent absence of Purkinje cell involvement. This variation among the ataxias makes it unclear as to the extent to which a therapy targeted to the DCN will be generally effective. Moreover, while riluzole is FDAapproved, the mechanism of its neuroprotective action in vivo is unknown. Cell-based studies, besides suggesting that riluzole may be an SK opener, have indicated that it inhibits glutamate release and blocks voltage-gated calcium channels, $\mathrm{Na}^{+}$channels, and voltage-gated $\mathrm{K}^{+}$channels, in addition to other activities. Based on its reported effect as an inhibitor of glutamate excitotoxicity, riluzole has undergone clinical trials for the treatment of amyotrophic lateral sclerosis. Unfortunately, the therapeutic benefit of riluzole in these studies has been only a very minimal improvement in prognosis for patients (9).

Overall, the results reported by Shakkottai et al. (4) provide genetic evidence that firmly supports the idea that, in vivo, the firing rate of DCN neurons is indeed regulated by SK channels. These data go a long way toward removing any doubt that may have existed as to the role of SK channels as regulators of DCN firing (10). Furthermore, even with a caveat that other brain regions may also contribute to the ataxia in the SK3-1B mice, it seems quite likely that a chronic enhancement of DCN firing rate can 
be a critical factor in the development of ataxia. Without question, their data do suggest that reestablishing a normal DCN firing rate might have therapeutic potential.

1. Ramón y Cajal, S. 1888. Sobre las fibras nerviosas de la capa molecular del cerebelo. Rev. Trim. Histol. Normal Patol. 1:33-49.

2. Kim, J.J., and Thompson, R.F. 1997. Cerebellar circuits and synaptic mechanisms involved in classical eyeblink conditioning. Trends Neurosci. 20:177-181

3. Heslow, G., and Ivarsson, M. 1994. Suppression of cerebellar Purkinje cells during conditioned responses in ferrets. Neuroreport. 5:649-652.

4. Shakkottai, V.G., et al. 2004. Enhanced neuronal excitability in the absence of neurodegeneration induces cerebellar ataxia. J. Clin. Invest. 113:582-590. doi:10.1172/JCI200420216.

5. Aizenman, C.D., and Linden, D.J. 1999. Regulation of the rebound depolarization and spontaneous firing patterns of deep nuclear neurons in slices of rat cerebellum. J. Neurophysiol. 82:1697-1709.

6. Tomita, H., et al. 2003. Novel truncated isoform of SK3 potassium channel is a potent dominantnegative regulator of SK currents: implications in schizophrenia. Mol. Psychiatry. 8:524-535.

7. Cao, Y.-J., Drexler, J.C., Couey, J.J., and Houamed, K.M. 2002. Modulation of recombinant and native neuronal SK channels by the neuroprotective drug riluzole. Eur. J. Pharmacol. 449:47-54. 8. Lowe, J.S., and Leigh, N. 2002. Disorders of movement and system degenerations. In Greenfield's neuropathology. Volume 2. 7th edition. D.I. Graham and P.L. Lantos, editors. Arnold Publishing. New York, New York, USA/London, United Kingdom. 325-430.

9. Festoff, B.W., Suo, Z., and Citron, B.A. 2003 Prospects for pharmacotherapy of amyotrophic lateral sclerosis: old strategies and new paradigms for the third millennium. CNS Drugs. 17:699-717.

10. Raman, I.M., Gustafson, A.E., and Padgett, D. 2000. Ionic currents and spontaneous firing in neurons isolated from the cerebellar nuclei. J. Neurosci. 20:9004-9016

\section{The eosinophil enigma}

\author{
Timothy J. Williams
}

School of Medicine, Imperial College London, London, United Kingdom

Eosinophils accumulate in high numbers in the lungs of asthmatic patients. These cells have the ability to induce tissue damage, a capacity that relates to their traditional role in host defense against parasitic worms. On the other hand, eosinophils produce growth factors associated with tissue repair and remodeling, notably TGF- $\beta_{1}$. The relationship of these activities to lung dysfunction in asthma is highly controversial, but recent observations in humans and in animal models add spice to the debate (see the related article beginning on page 551).

J. Clin. Invest. 113:507-509 (2004). doi:10.1172/JCI200421073.

Since the discovery of the eosinophil, a leukocyte with a granular cytoplasm that stains pink with eosin dye, the function of this cell has been disputed; opinions on its role have swung periodically, as it has been characterized alternately as damaging and protective. Eosinophils accumulate in tissues in response to parasitic worm infections and, on activation, release cationic proteins from their granules and reactive oxygen species that are toxic to worms. Allergy may be an aberration of this defense process whereby otherwise innocuous agents in the environment stimulate an

\footnotetext{
Address correspondence to: Timothy J. Williams, Leukocyte Biology Section, Biomedical Sciences Division, School of Medicine, Imperial College London, Sir Alexander Fleming Building, Exhibition Road, London SW7 2AZ, United Kingdom. Phone: 44-020-7594-3159;

Fax: 44-020-7594-3119;

E-mail: tim.williams@imperial.ac.uk.

Conflict of interest: The author has declared that no conflict of interest exists.

Nonstandard abbreviations used:

bronchoalveolar lavage fluid (BALF).
}

adaptive immune response, characteristically driven by Th2 lymphocytes, resulting in the recruitment and activation of eosinophils that induce tissue damage. Eosinophils also release leukotrienes that induce smooth muscle contraction and microvascular leakage. IL-5, produced by Th2 lymphocytes, is critically important in this process, as it mediates the terminal differentiation and proliferation of eosinophils in the bone marrow and their release into the blood. IL-5 also primes eosinophils, for example, to facilitate eosinophil recruitment into tissues mediated by chemokines such as eotaxins, and IL-5 also prolongs eosinophil survival.

The key role of IL-5 in eosinophil production and function has made this cytokine a prime therapeutic target. This has been reinforced by results in animal models of asthma showing that deletion of the IL-5 gene (1) or the use of IL-5-neutralizing antibodies (2) is able to suppress eosinophil recruitment to the lung and, in many but not all cases (3), inhibit hyper-responsiveness of the airways to agents that induce bronchoconstriction, a characteristic feature of allergic asthma. Double eotaxin-1/IL-5 gene-deleted mice exhibited a greater suppression of eosinophil recruitment to the lung and the abolition of airway hyper-responsiveness associated, interestingly, with reduced IL-13 production (4).

Animal studies, particularly those in allergic cynomolgus monkeys showing long-term inhibition of lung eosinophilia and airway hyperresponsiveness by a single dose of an anti-IL-5 antibody (5), encouraged the initiation of clinical trials in which antibody was administered to mild atopic asthmatics (6) and chronic severe asthmatics (7). These studies showed no consistent effects on lung function tests, despite a marked suppression of blood eosinophils. From the results of these studies, some concluded that eosinophils are not causally related to lung dysfunction, although the results are contentious, particularly because of the small size of the trials and their technical limitations $(8,9)$. Furthermore, a followup study of bronchial biopsies from antibody-treated patients showed that despite marked depletion of blood and sputum eosinophils, airway tissue eosinophils were only depleted by $55 \%$ (10), suggesting that the residual cells could be important in lung dysfunction.

\section{The eosinophil in tissue remodeling}

The finding that eosinophils are able to produce growth factors directed attention to the potential role of these cells in the resolution of inflammation 